http://jmscr.igmpublication.org/home/ ISSN (e)-2347-176x ISSN (p) 2455-0450

crossref DOI: https://dx.doi.org/10.18535/jmscr/v8i11.21

\author{
Journal Of Medical Science And Clinical Research \\ IGM Publication \\ An Official Publication of IGM Publication
}

\title{
Case Report on Malignant Mixed Mullerian Tumor, Endometrium with heterologous elements
}

\author{
Authors \\ Dr Akshita Mehta ${ }^{1}$, Dr Sarita Asotra ${ }^{2 *}$ \\ ${ }^{1}$ Junior Resident, Department of Pathology, IGMC Shimla, Himachal Pradesh \\ ${ }^{2}$ Associate Professor, Department of Pathology, IGMC Shimla, H.P. \\ *Corresponding Author \\ Dr Sarita Asotra \\ Associate Professor, Department of Pathology, IGMC SHIMLA, H.P., India
}

\begin{abstract}
Malignat mixed mullerian tumor of endometrium also called carcinosarcoma. MMMT is a rare and aggressive form of cancer of uterus, the ovaries, the fallopian tubes and other parts of the body with malignant epithelial as well as mesenchymal component. We present a case of 70 year old post-menopausal women with complaints of post-menopausal bleeding and pain abdomen treated with hysterectomy and bilateral salpingo-oophorectomy histopathologically diagnosed with Carcinosarcoma.
\end{abstract}

\section{Introduction}

Malignant mixed Mullerian tumor/ Carcinosarcoma of the uterus is rare, high-grade and aggressive neoplasms accounting for $<5 \%$ of tumors derived from the body of the uterus. ${ }^{1}$ These tumors are most commonly seen in postmenopausal females. ${ }^{2}$ It is divided into two types, homologous, in which the sarcomatous component is made of tissues found in the uterus such as endometrial, fibrous and/or smooth muscle tissues and a heterologous type which is made up of tissues not found in the uterus, such as cartilage, skeletal muscle or bone . Risk factors include obesity, exogenous estrogen therapies, nulliparity, tamoxifen therapy and pelvic irradiation. $^{3}$

\section{Case Report}

70 years old post-menopausal female presented with pain abdomen from last 8 months and intermittent bleeding per vaginum from 5-6 months. She is P4L4 with history of tubectomy 27 years ago and is menopausal from last 8 years. There is also history of mastectomy done in 2005 for Infiltrating Ductal cell Carcinoma, left breast and was on tamoxifen therapy till 2015.

On transabdominal utrasonography of the patient bulky uterus was noted along with thickened endometrium and clinico-radiological diagnosis of Endometrial Carcinoma was proposed. She underwent abdominal hysterectomy with bilateral salpingo-oophorectomy.

The gross specimen showed an already cut open uterus with cervix $\left(8^{*} 6^{*} 3\right) \mathrm{cms}$ and grossly unremarkable bilateral adnexa. There is a graywhite, solid, friable growth in endometrial cavity $(4 * 6 * 2) \mathrm{cms}$ extending grossly upto outer onethird of the myometrium. 


\section{JMSCR Vol||08||Issue |11||Page 119-122||November}

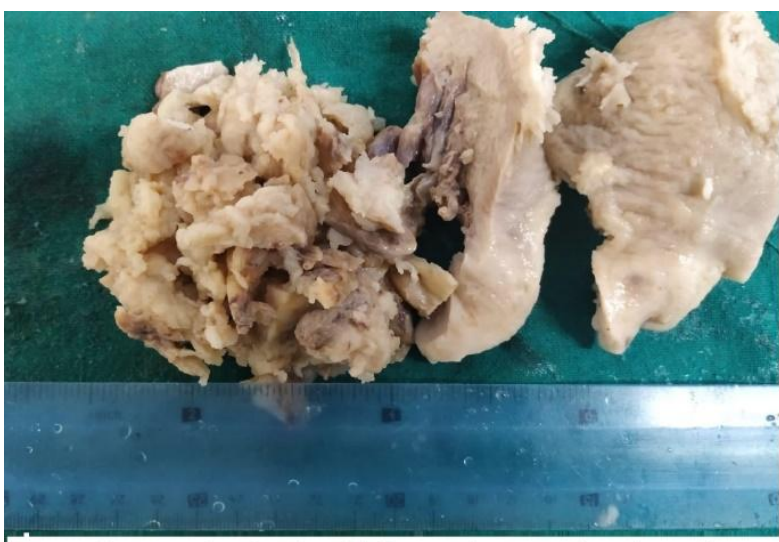

Gross photograph of polypoidal growth occupying the entire endometrial cavity

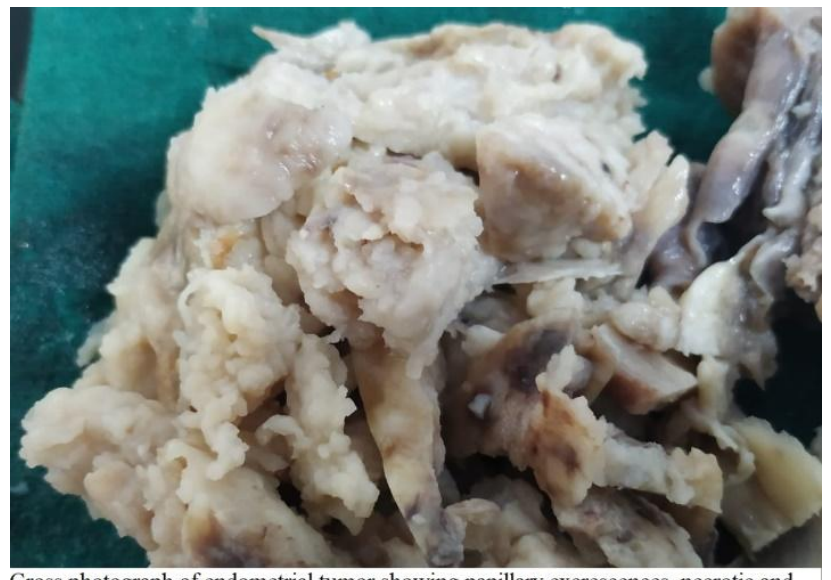

Gross photograph of endometrial tumor showing papillary excrescences, necrotic and hemorrhagic areas

Microscopic examination of sections from tumor showed biphasic morphology with epithelial component showing tumor cells arranged in papillae, trabeculae and cyst having moderate pleomorphism with clear cell change, increased mitosis and hob nailing. Mesenchymal component revealed ovoid to spindle tumor cells (endometrial stromal sarcoma) and heterologous element of Chondrosarcoma. Additionally, focal areas of myxoid change, psammoma bodies seen.

Tumor extending upto inner half of the myometrium and involving one side fallopian tube. Cervix, other side fallopian tube and bilateral ovaries unremarkable.
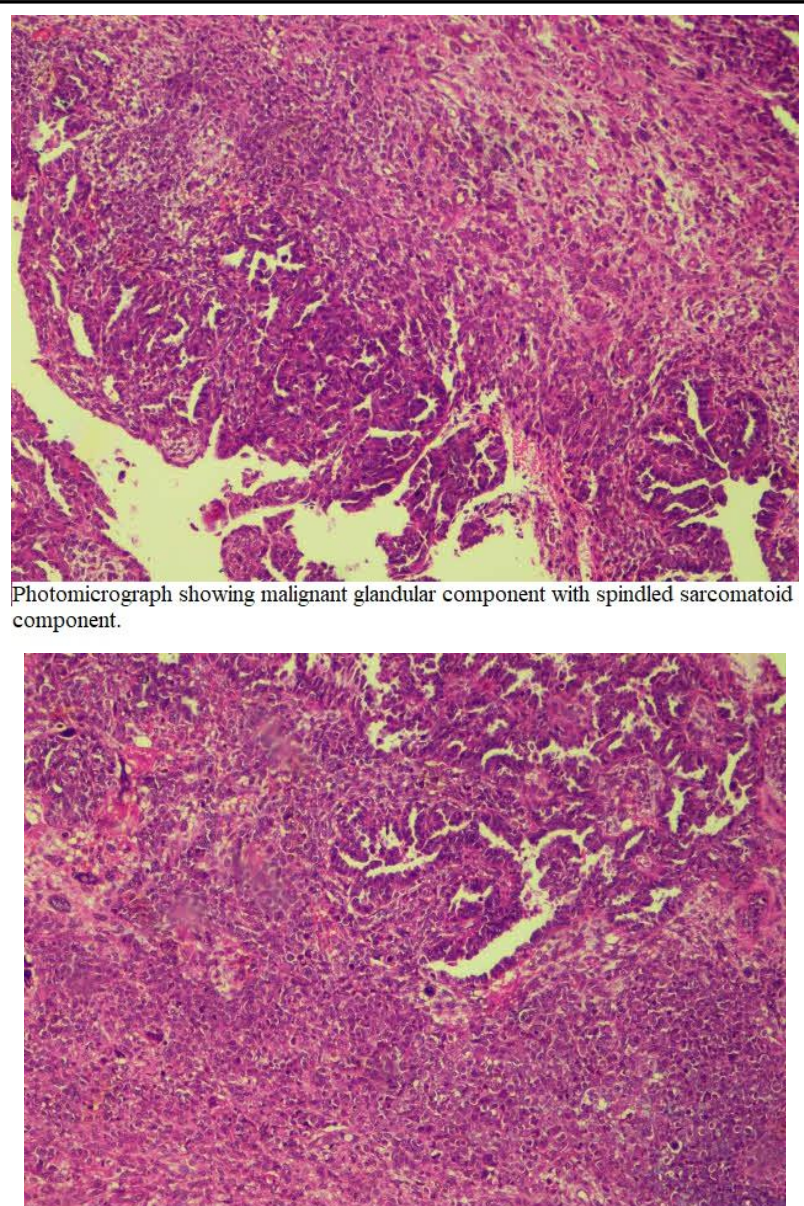

Photomicrograph showing carcinosarcoma of endometrium with both carcinomatous and sarcomatous component having malignant cytological feature.

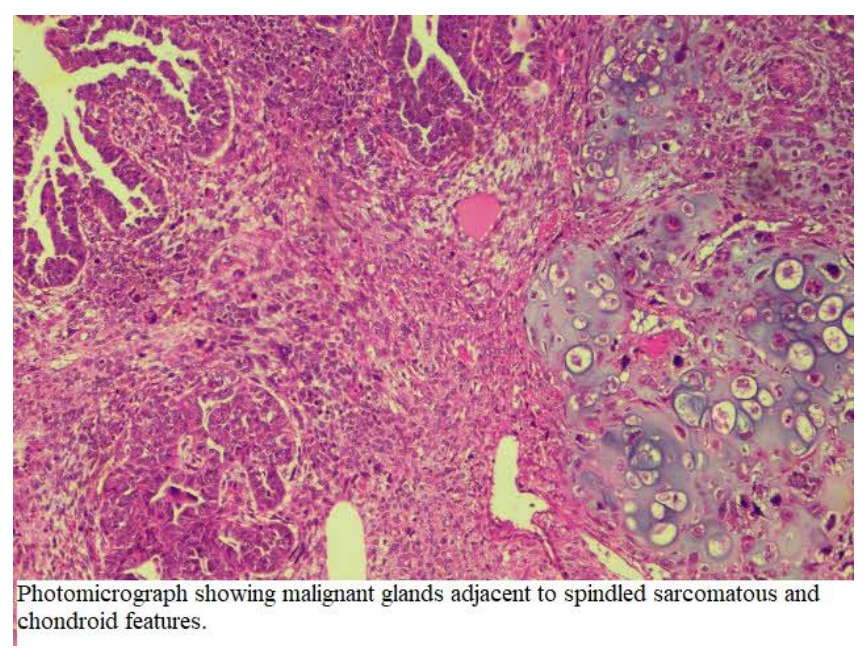

Histopathological diagnosis of Malignant Mixed Mullerian tumor, endometrium with endometroid/ clear cell carcinoma and endometrial stromal sarcoma with heterologous elelment of Chondrosarcoma was given.

On IHC the glandular element was immunoreactive for CK, ER. P53 and ki67 while the stromal component was immunoreactive for CK, CD10, Cyclin-D1, p53 and ki67 


\section{Discussion}

MMMT is rare aggressive tumor of uterus and can also occur in different parts of body. The incidence of MMT has been identified in decreasing order of frequency in the vagina, cervix, and ovary and most rarely in the fallopian tubes. $^{4}$

There are three main theories regarding the histogenesis of MMMT namely ${ }^{5}$ :

The collision theory suggests that the carcinoma and sarcoma are two independent neoplasms.

The combination theory suggest that both components and derived from a single stem cell that undergoes divergent differentiation early in the evolution of the tumor.

The conversion theory suggests that the sarcomatous elements derived from the carcinoma during the evolution of the tumor.

Among risk factors, most important attributable to MMMT is estrogen excess and Tamoxifen therapy. Depending on the target organs, tamoxifen exerts both an estrogenic and an antiestrogenic effect. Tamoxifen has an estrogenic effect on the uterus, bone and liver. It can induce proliferation of endometrial glands and periglandular stromal condensation due to the presence of ERs in the glandular epithelium and stromal cells.

Tamoxifen, additionally induces genes producing adrenomedullin, a growth factor for endothelial cells that upregulates bcl-2 and enhances the survival of malignant cells under hypoxic conditions. ${ }^{6}$

Treilleux et $a l,{ }^{7}$ the relative risk of developing endometrial cancer in cases of early breast cancer was significantly reduced when the dose of tamoxifen was halved

Extension to pelvis, lymphatic and vascular permeation, distant lymph node, and blood-borne metastasis are common. In our case, there was no evidence of lymph node or distant metastasis. The average 5-year survival was 21\%; 70-90\% of tumor- related deaths occurred within 18 months of diagnosis ${ }^{8}$. The most important prognostic factor is the extent of the tumor at the time of diagnosis. Some authors have described that malignant mixed Mullerian tumors with heterologous elements are worse than those with the homologous components. ${ }^{8}$

\section{Conclusion}

Malignant Mixed Mullerian Tumor is a rare and aggressive tumor. It has association with tamoxifen therapy which is seen in our case. Our case was of Hetrologous MMMT which is considered more aggressive. Outcome depends on type of component (homologous/heterologous) and the extent of tumor. Treatment is surgery along with radiotherapy and chemotherapy

\section{Reference}

1. Tekwani DT, Joshi SR, Pathak S, Nagare M, Bihade A, Kendre D. Malignant mixed mullerian tumor of the uterus: A case report. Ind J Basic Appl Medl Res 2013;3:33-36.

2. Thawal YA, Tambe SG, Tania A, Chavan RR, Patel JA. A rare case of malignant mixed mullerian tumour of uterine corpus. Int J Med Appl Sci 2014;3:100-102.

3. Brooks SE, Zhan M, Cote T, Baquet CR. Surveillance, epidemiology, and end results analysis of 2677 cases of uterine sarcoma 1989-1999. Gynecol Oncol. 2004; 93:204-208.

4. Ahuja A, Safaya R, Prakash G, Kumar L, Shukla, NK. Primary mixed Mullerian tumor of the vagina: a case report with review of the literature. Pathology Research and Practice. 2011;207(4):253255

5. Jin Z, Ogata S, Tamura G. Carcinosarcomas (malignant Mullerian mixed tumors) of the uterus and ovary: a genetic study with special reference to histogenesis. Int $\mathbf{J}$ Gynecol Pathol. 2003;22(4):368- 373.

6. Zhao Y, Hague S, Manek S et al. . PCR display identifies tamoxifen induction of the novel angiogenic factor 
adrenomedullin by a nonestrogenic mechanism in the human endometrium. Oncogene 1998;16:409-415.

7. Treilleux $\mathrm{T}$, Mignotte $\mathrm{H}$, ClementChassagne $\mathrm{C}$ et al. Tamoxifen and malignant epithelial- nonepithelial tumours of the endometrium: report of six cases and review of the literature. Eur $\mathbf{J}$ Surg Oncol 1999;25:477-482.

8. Barani K, Padmavathi R. Two rare cases of malignant mixed mullerian tumor. Internet J Pathol 2013; 15:1-8. 